\title{
GENERALIZED INTERSECTION MULTIPLICITIES OF MODULES
}

BY

SANKAR P. DUTTA

\begin{abstract}
In this paper we study intersection multiplicities of modules as defined by Serre and prove that over regular local rings of $\operatorname{dim} \leqslant 5$, given two modules $M, N$ with $l\left(M \otimes_{R} N\right)<\infty$ and $\operatorname{dim} M+\operatorname{dim} N<\operatorname{dim} R, \chi(M, N)=$ $\sum_{i=0}^{\operatorname{dim}} R(-1)^{i} l\left(\operatorname{Tor}_{i}^{R}(M, N)\right)=0$. We also study multiplicity in a more general set up. Finally we extend Serre's result from pairs of modules to pairs of finite free complexes whose homologies are killed by $I^{n}, J^{n}$, respectively, for some $n>0$, with $\operatorname{dim} R / I+\operatorname{dim} R / J<\operatorname{dim} R$.
\end{abstract}

In Algèbre locale. Multiplicités [S] Serre has proved the following theorem: If $R$ is an equicharacteristic or unramified regular local ring and $M, N$ are two finitely generated modules over $R$ such that $l\left(M \otimes_{R} N\right)<\infty$, then

$$
\chi(M, N)=\sum_{i=0}^{\operatorname{dim} R}(-1)^{i} l\left(\operatorname{Tor}_{i}^{R}(M, N)\right) \geqslant 0
$$

and $=0$ if and only if $\operatorname{dim} M+\operatorname{dim} N<\operatorname{dim} R$. In the same book Serre conjectured that the above result is true for any regular local ring. In 1970/71 M. P. Mallaivain-Brameret $[\mathbf{M}]$ proved the special case of Serre's conjecture in the ramified case (i.e. $p \in m^{2}$ ) when $p M=0, p N=0$. In 1972, M. Hochster [H] proved Serre's conjecture for regular local rings with dimension $\leqslant 4$. In 1975 Peskine and Szpiro proved the conjecture for graded modules over graded rings $R$ with $R$ finitely generated over $R_{0}$ and $R_{0}$ is Artinian.

In this paper we start with a greater generality: Let $\mathcal{C}$ be the set of pairs of modules $(M, N)$ over a C-M ring $R$ such that $l\left(M \otimes_{R} N\right)<\infty, \operatorname{pd} M<\infty, \operatorname{dim} M$ $+\operatorname{dim} N \leqslant \operatorname{dim} R$. We say that $R$ satisfies the vanishing conjecture if $\chi(M, N)=0$ whenever $(M, N) \in \mathcal{C}$ and $\operatorname{dim} M+\operatorname{dim} N<\operatorname{dim} R$, and that $R$ satisfies the nonvanishing conjecture if $\chi(M, N)>0$ whenever $(M, N) \in \mathcal{C}$, and $\operatorname{dim} M+$ $\operatorname{dim} N=\operatorname{dim} R$.

In this work, all rings are noetherian local with identity and all modules are finitely generated.

We show in (1.1) that the vanishing conjecture holds for two-dimensional C-M rings. Using this fact we prove in 1.4 Serre's Conjecture for the pair $(M, N) \in \mathcal{C}$, $\operatorname{dim} N=1$ over a regular local ring and also give another proof that the above result implies vanishing for regular local rings $R$ with $\operatorname{dim} R \leqslant 4$. (M. Hochster proved this

Received by the editors March 29, 1982.

1980 Mathematics Subject Classification. Primary 13H15; Secondary 13D99, 14C17. 
in 1972. See [H]. Recently H. B. Foxby has proved the case of $\operatorname{dim} N=1$ in a more general set-up. See [F].) In 2.4 we show that the vanishing conjecture holds on a Gorenstein ring $R$ if and only if for all pairs $(M, N) \in \mathcal{C}$, with $M$ perfect, $N$ C-M, $\operatorname{dim} M+\operatorname{dim} N=\operatorname{dim} R, l\left(M \otimes_{R} N\right)=l\left(M \otimes_{R} N\right)$. Using this criterion we prove in 2.5 that over a regular local ring of dimension 5 the vanishing conjecture holds. $\S \S 2.4,2.6$, and 2.7 provide some special cases of the vanishing conjecture. $\$ 3.1$ establishes that the vanishing conjecture holds if and only if for all pairs $(M, N) \in \mathcal{C}$, with pd $M<\infty, \operatorname{dim} M+\operatorname{dim} N<\operatorname{dim} R, \chi_{1}(M, N)>0$ and, prolonging this argument in 3.2 , we show that the nonvanishing conjecture implies the vanishing conjecture. In 4.1 and 4.2 we extend the vanishing part of Serre's theorem to pairs of finite free complexes $C, C^{\prime} \operatorname{such}$ that $\operatorname{dim}(R / I)+\operatorname{dim}(R / J)<\operatorname{dim} R$, $l(R /(I+J))<\infty$, where $I=\mathrm{Ann}_{R} H(C), J=\mathrm{Ann}_{R} H\left(C^{\prime}\right)$, and $R$ is regular. Most of the main results of this paper were obtained when I was doing my Ph.D. thesis under the guidance of M. Hochster at the University of Michigan. I would like to thank him for his constant inspiration and encouragement throughout this work.

List of abbreviations and notations and a special lemma.

$$
\begin{aligned}
l(M) & =\text { length of the module } M, \\
Q & =\text { the field of rational numbers, } \\
\text { C-M } & =\text { Cohen-Macaulay, } \\
\text { ch } p & =\text { characteristic } p \\
\text { n.z.d. } & =\text { nonzero divisor, } \\
\text { d.v.r. } & =\text { distance valuation ring, } \\
Q\{R\} & =\text { total quotient ring of the ring } R, \\
\text { pd } M & =\text { projective dimension of } M, \\
r(N) & =\text { torsion-free rank of } N, \\
Q\{N\} & =N \otimes_{R} Q\{R\}, \\
p^{(n)} & =\left\{x \in R \mid t x \in p^{n} \text { for some } t \in R-p\right\} .
\end{aligned}
$$

Let $R$ be a local ring with 1 . Let $M$ be a finitely generated module with finite projective dimension $n$. Let $N$ be another finitely generated module with $l(M \otimes N)$ $<\infty$. Then

$$
\chi_{i}(M, N)=\sum_{k=0}^{n-i}(-1)^{k} l\left(\operatorname{Tor}_{i+k}^{R}(M, N)\right) .
$$

The following lemma has been used many times in this work. We leave the proof as an exercise for the reader.

S. L. (SPECIAL LemMA). Let $R$ be a local ring with 1 . Let $M$ be a finitely generated module with pd $M=n$. Let $N$ be another module with $M \otimes_{R} N \neq 0$ and let $\mathrm{Ann}_{R} M$ contain an $N$-sequence of length $r$. Then $\operatorname{Tor}_{n-i}^{R}(M, N)=0,0 \leqslant i<r$.

We also state two results from [D1, 2.2] which have been referred to many times in this work. 
LEMMA 1. Let $R$ be Gorenstein of $\operatorname{dim} n$. For any two modules $M, N$ with pd $M<\infty, l\left(M \otimes_{R} N\right)<\infty$ and $\operatorname{dim} M+\operatorname{dim} N<\operatorname{dim} R, \chi(M, N)=0$ if and only if for any perfect module $M, C-M$ module $N$ such that $l\left(M \otimes_{R} N\right)<\infty$ and $\operatorname{dim} M+\operatorname{dim} N=n-1, \chi(M, N)=0$.

Claim 1. Let $M$ and $N$ be two modules over a C-M ring such that $l\left(M \otimes_{R} N\right)<\infty$ and $\operatorname{dim} M+\operatorname{dim} N<\operatorname{dim} R$. Then we can choose a system of parameters $\left\{x_{1}, \ldots, x_{r}\right\}$ for $M$ contained in $\mathrm{Ann}_{R} N$ such that $\left\{x_{1}, \ldots, x_{r}\right\}$ is an $R$-sequence where $r=\operatorname{dim} M$.

1.

1.1 Proposition. Let $R$ be a $C$-M local ring and $\operatorname{dim} R=2$. Let $M$ be a module of finite length and finite projective dimension over $R$. Let $N$ be another module over $R$ with $\operatorname{dim} N \leqslant 1$. Then $\chi(M, N)=0$.

Proof. First we prove the following lemma.

LEMMA. Let $R$ be a commutative ring with 1 . If $0 \rightarrow R^{n_{k}} \rightarrow \cdots \rightarrow R^{n_{0}} \rightarrow M \rightarrow 0$ is exact, $x \in R$ is not nilpotent in $R$ and $x$ is nilpotent on $M$, then $\sum_{i=0}^{k}(-1)^{i} n_{i}=0$.

Proof. Since $x$ is nilpotent on $M, M \otimes_{R} R_{x}=0$. Thus the result follows by applying $\otimes_{R} R_{x}$ to the given exact sequence. Now we return to the proof of the theorem.

Since $l(M)<\infty$, pd $M<\infty$ and depth $R=2$, from $\operatorname{depth} M+\operatorname{pd} M=$ depth $R$, we get pd $M=2$. Let $k=R / m$, where $m$ is the maximal ideal of $R$. Let

$$
0 \rightarrow R^{n_{2}} \rightarrow R^{n_{1}} \rightarrow R^{n_{0}} \rightarrow M \rightarrow 0
$$

be a minimal projective resolution of $M$. Then

$$
\chi(k, M)=\sum_{i=0}^{2}(-1)^{i} n_{i}=0
$$

(by the above lemma). When $\operatorname{dim} N=0, l(N)<\infty$, hence by taking a filtration of $N$ with all factors isomorphic to $R / m$, and noting the fact that, given $0 \rightarrow N_{1} \rightarrow N_{2}$ $\rightarrow N_{3} \rightarrow 0$ is exact,

$$
\chi\left(N_{2}, M\right)=\chi\left(N_{1}, M\right)+\chi\left(N_{3}, M\right)
$$

we get

$$
\chi(N, M)=l(N) \chi(k, M)=0 .
$$

Next we consider the case when $\operatorname{dim} N=1$. Since any filtration of $R / p^{n}$ contains $l\left(R_{p} / p^{n} R_{p}\right)$ copies of $R / p$ and each of the remaining quotients in the filtration is isomorphic to $R / m$, we have by (3) and (4),

$$
\chi\left(R / p^{n}, M\right)=l\left(R_{p} / p^{n} R_{p}\right) \chi(R / p, M),
$$

i.e.

$$
\chi(R / p, M)=\chi\left(R / p^{n}, M\right) / l\left(R_{p} / p^{n} R_{p}\right)
$$


Now we consider the exact sequence

$$
0 \rightarrow p^{(n)} / p^{n} \rightarrow R / p^{n} \rightarrow R / p^{(n)} \rightarrow 0 .
$$

Since $\operatorname{dim} R / p^{n}=1, \operatorname{dim} p^{(n)} / p^{n}=0$, i.e., $p^{(n)} / p^{n}$ is a module of finite length. Hence by (4),

$$
\chi\left(R / p^{n}, M\right)=\chi\left(R / p^{(n)}, M\right)
$$

and by (5),

$$
\chi(R / p, M)=\lim _{n \rightarrow \infty} \frac{\chi\left(R / p^{(n)}, M\right)}{l\left(R_{p} / p^{n} R_{p}\right)} .
$$

Since $\operatorname{dim} R_{p}=1, l\left(R_{p} / p^{n} R_{p}\right)$ is a linear polynomial in $n$ for $n>0$. Thus we will be done if we can show

$$
\lim _{n \rightarrow \infty} \frac{\chi\left(R / p^{(n)}, M\right)}{n}=0 .
$$

Since $R / p^{(n)}$ has depth 1 and Ann $M$ is $m$-primary, Ann $M$ contains a $R / p^{(n)}$ regular element. Therefore, by Lemma (S.L.), $\operatorname{Tor}_{2}^{R}\left(R / p^{(n)}, M\right)=0$. So

$$
\chi\left(R / p^{(n)}, M\right)=l\left(M / p^{(n)} M\right)-l\left(\operatorname{Tor}_{1}^{R}\left(R / p^{(n)}, M\right)\right) .
$$

Since $l\left(M / p^{(n)} M\right)<l(M)$,

$$
\lim _{n \rightarrow \infty} \frac{1}{n} l\left(M / p^{(n)} M\right)=0 .
$$

Since $l(M)<\infty$, we have the exact sequence

$$
0 \rightarrow M \rightarrow\left(R /\left(x_{1}, x_{2}\right)\right)^{t} \rightarrow Q \rightarrow 0
$$

where $\left\{x_{1}, x_{2}\right\}$ is an $R$-sequence. By Lemma (S.L.) $\operatorname{Tor}_{2}^{R}\left(R / p^{(n)}, R\left(x_{1}, x_{2}\right)\right)=0$; hence $0=\chi\left(R / p^{(n)}, R /\left(x_{1}, x_{2}\right)\right)$. (Since $l\left(R /\left(x_{1}, x_{2}\right)\right)<\infty$ and $\left\{x_{1}, x_{2}\right\}$ is an $R$-sequence,

$$
0=l\left(R /\left\{p^{(n)}+\left(x_{1}, x_{2}\right)\right\}\right)-l\left(\operatorname{Tor}_{1}^{R}\left(R / p^{(n)}, R /\left(x_{1}, x_{2}\right)\right)\right),
$$

i.e.,

$$
l\left(\operatorname{Tor}_{1}^{R}\left(\frac{R}{p^{(n)}}, \frac{R}{\left(x_{1}, x_{2}\right)}\right)\right)=l\left(\frac{R}{p^{(n)}+\left(x_{1}, x_{2}\right)}\right)<l\left(\frac{R}{\left(x_{1}, x_{2}\right)}\right) .
$$

Since $l(Q)<\infty, \operatorname{pd}(Q)<\infty$, by Lemma (S.L.), $\operatorname{Tor}_{2}^{R}\left(R / p^{(n)}, Q\right)=0$. Thus applying $\otimes R / p^{(n)}$ to (12) we get

$$
0 \rightarrow \operatorname{Tor}_{1}^{R}\left(\frac{R}{p^{(n)}}, M\right) \rightarrow \operatorname{Tor}_{1}^{R}\left(\frac{R}{p^{(n)}},\left(\frac{R}{\left(x_{1}, x_{2}\right)}\right)^{t}\right) \rightarrow \cdots
$$

exact and hence

$$
l\left(\operatorname{Tor}_{1}^{R}\left(\frac{R}{p^{(n)}}, M\right)\right) \leqslant l\left(\operatorname{Tor}_{1}^{R}\left(\frac{R}{p^{(n)}},\left(\frac{R}{\left(x_{1}, x_{2}\right)}\right)^{t}\right)\right) \leqslant t l\left(\frac{R}{\left(x_{1}, x_{2}\right)}\right)
$$


by (13) which shows

$$
\lim _{n \rightarrow \infty} \frac{1}{n} l\left(\operatorname{Tor}_{1}^{R}\left(\frac{R}{p^{(n)}}, M\right)\right)=0 .
$$

Now by (9), (10), (11), and (14) we are done.

1.2. M. Hochster has proved Serre's multiplicity conjecture for regular local rings $R$ with $\operatorname{dim} R \leqslant 4$ (see [H]). To prove the vanishing part he has used the fact that after extending the ring the Grothendieck group of modules of finite length and finite projective dimension is generated by modules of the type $R /\left(x_{1}, x_{2}\right)$, where $\left\{x_{1}, x_{2}\right\}$ is an $R$-sequence, $\operatorname{dim} R=2$. By using 1.1 we can give another proof of the vanishing part in the following way:

We have to show that given $M, N$ on $R$, where $\operatorname{dim} R \leqslant 4, \operatorname{dim} M+\operatorname{dim} N<$ $\operatorname{dim} R$, and $l(M \otimes N)<\infty$, then $\chi(M, N)=0$. We note that for $\operatorname{dim} R=1$ the proof is obvious, $\operatorname{dim} R=2$ is done in 1.1. So we consider the cases where $\operatorname{dim} R=3$ or 4 . We can assume $M, N$ are both C-M and $\operatorname{dim} M+\operatorname{dim} N=2$ (or 3) [D1, 2.2]. Without loss of generality we may suppose $\operatorname{dim} N=1$ (or 2). By [D1, 2.2] we are reduced to proving the vanishing conjecture over C-M rings of $\operatorname{dim} 2$ which was already done in 1.1 .

1.3 Proposition. Let $R$ be a $C-M$ ring of $\operatorname{dim} n$. Let $N$ be a module of $\operatorname{dim} 1$ and $M$ a module of finite projective dimension with $\operatorname{dim} M \leqslant n-2$. Then $\chi(M, N)=0$.

Proof. By [D1] we can assume without any loss of generality that $M$ is perfect, $\operatorname{dim} M=n-2$. Again by [D1, 2.2] we are reduced to proving the vanishing conjecture on a 2-dimensional C-M ring. Thus we are done by 1.1 .

1.4 TheOREM. Let $N$ be a module with $\operatorname{dim} N=1$ over a regular local ring $R$. Let $M$ be another module such that $l\left(M \otimes_{R} N\right)<\infty$. Then

(i) $\operatorname{dim} M+\operatorname{dim} N<\operatorname{dim} R$ implies $\chi(M, N)=0$;

(ii) $\operatorname{dim} M+\operatorname{dim} N=\operatorname{dim} R$ implies $\chi(M, N)>0$.

(Since $R$ is regular, $\operatorname{dim} M+\operatorname{dim} N \leqslant \operatorname{dim} R$.)

Proof. (ii) $\operatorname{dim} N=1$ implies $\operatorname{dim} M=n-1$ where $n=\operatorname{dim} R$. Since $\operatorname{dim} M=$ $n-1$, the minimal primes of $\operatorname{Ass}_{R}(M)$ are primes $p_{i}$ of ht 1, i.e., $p_{i}=\left(x_{i}\right)$ for some prime element $x_{i} \in R$. Thus by taking a prime filtration of $M$ by (3), 1.1, we get

$$
\chi(M, N)=\sum \chi\left(N, R /\left(x_{i}\right)\right)+\sum_{\text {ht } q_{j} \geqslant 2} \chi\left(N, R / q_{j}\right) .
$$

Since $\operatorname{dim} N=1, \chi\left(N, R /\left(x_{i}\right)\right)>0$ by [L, Theorem 1]. Hence if we show $\chi(N, Q)$ $=0$ with $\operatorname{dim} Q \leqslant n-2$, by (1) we will be done. Hence the proof of (ii) now reduces to (i).

(i) By $[\mathrm{D1}, 2.2]$ we can assume $M$ is perfect, $\operatorname{dim} M=n-2$. Therefore we are done by the two previous propositions.

2.

2.1 Definition. Let $R$ be a C-M ring of dimension $n$ with canonical module $\Omega$ and let $M$ be a C-M module of dimension $d$. We define $\check{M}$ to be $\operatorname{Ext}_{R}^{n-d}(M, \Omega)$. 
Proposition. Let $R$ be a Gorenstein ring of $\operatorname{dim} n$. Let $M$ be a perfect module and let $N$ be $C$ - $M$ such that (i) $\operatorname{dim} M+\operatorname{dim} N=n$; (ii) $l(M \otimes N)<\infty$. Then $l(M \otimes N)$ $=l(\check{M} \otimes \check{N})$.

Proof. Let $\operatorname{dim} M=r, \operatorname{dim} N=s$. Since $M$ is perfect, $\operatorname{pd} M=n-r=s$. Since $R$ is Gorenstein, $\Omega_{R}=$ canonical module of $R \simeq R$. We have by local duality $\operatorname{Ext}^{i}(M, R)=0, i \neq s, \operatorname{Ext}^{s}(M, \Omega) \neq 0 ; \operatorname{Ext}^{j}(N, R)=0, j \neq r, \operatorname{Ext}^{r}(N, R) \neq 0$ (since both $M$ and $N$ are C-M). We note that if $l(T)<\infty$, for any module $T$, then $l(T)=l(\check{T})$. We have

$$
\begin{aligned}
l(M \otimes N) & =l(M \check{\otimes} N), \\
(M \ddot{\otimes} N) & =\operatorname{Ext}^{n}(M \otimes N, R) .
\end{aligned}
$$

Since $l(M \otimes N)<\infty$, by Claim 1, Lemma 1 ([D1, 2.2]), Ann $M$ contains a maximal $N$ sequence. Hence $\operatorname{Tor}_{i}^{R}(M, N)=0$ for all $i \neq 0$. We know both the spectral sequences $\operatorname{Ext}^{i}\left(\operatorname{Tor}_{j}(M, N), R\right)$ and $\operatorname{Ext}^{i}\left(M, \operatorname{Ext}^{j}(N, R)\right)$ converge to the same limit. When $i+j=n$ each degenerates and hence we have

$$
\operatorname{Ext}^{n}(M \otimes N, R) \simeq \operatorname{Ext}^{s}\left(M, \operatorname{Ext}^{r}(N, R)\right) .
$$

Since pd $M=s$ and $\operatorname{Ext}^{i}(M, R)=0, i<s$, we have from (3),

$$
\operatorname{Ext}^{s}\left(M, \operatorname{Ext}^{r}(N, R)\right) \simeq \check{M} \otimes \check{N} .
$$

From (1)-(4) the required result follows.

2.2 TheOReM. Let $R$ be a Gorenstein ring of $\operatorname{dim} n$. Let $M$ be perfect and let $N$ be $C-M$ such that $l\left(M \otimes_{R} N\right)<\infty$. Suppose $\operatorname{dim} M+\operatorname{dim} N \leqslant \operatorname{dim} R$. Let $i=n-$ $\operatorname{dim} M-\operatorname{dim} N$. Then $\chi(M, N)=(-1)^{i} \chi(\check{M}, \check{N})$.

Proof. First we note the following facts:

(A) If $R$ is Gorenstein local and $M$ is perfect, $N$ is any finitely generated module,

$$
\operatorname{Tor}_{j}^{R}(M, N) \simeq \operatorname{Ext}^{\mathrm{pd} M-i}(\check{M}, N) .
$$

Also the spectral sequences $\operatorname{Ext}^{p}\left(M, \operatorname{Ext}^{q}(N, R)\right)$ and $\operatorname{Ext}^{i}\left(\operatorname{Tor}_{j}(M, N), R\right)$ with $p+q=i+j$, converge to the same limit.

(B) If $N$ is $\mathrm{C}-\mathrm{M}$ of $\operatorname{dim} r$ and $l(M \otimes N)<\infty$ the left spectral sequence degenerates (only $q=n-s$ gives a nonzero term) and so does the right $\left(\operatorname{all}^{\operatorname{Tor}_{j}}(M, N)\right.$ have finite length, so the Ext is 0 unless $i=n)$. Hence we get

$$
\operatorname{Ext}^{j+s}(M, \check{N})=\operatorname{Ext}^{n}\left(\operatorname{Tor}_{j}(M, N), R\right)=\operatorname{Tor}_{j}(M, N)^{\nu} .
$$

Thus if $R$ is Gorenstein, $M$ is perfect, $N$ is C-M and $l(M \otimes N)<\infty$,

$$
\begin{aligned}
\operatorname{Tor}_{j}(M, N) & \simeq \operatorname{Ext}^{j+s}(M, \check{N})^{\swarrow} \quad(\text { dual of }(\mathrm{B})) \\
& \simeq \operatorname{Tor}_{\mathrm{pd} M-(j+s)}(\check{M}, \check{N})^{\swarrow} \quad(\text { by }(\mathrm{A})) .
\end{aligned}
$$

Hence

$$
\begin{aligned}
\chi(M, N) & =\sum(-1)^{j} l\left(\operatorname{Tor}_{j}(M, N)\right)=\sum(-1)^{-j} l\left(\operatorname{Tor}_{\mathrm{pd} M-j-s}(M, N)\right) \\
& =(-1)^{-\mathrm{pd} M+s} \sum(-1)^{\mathrm{pd} M-s-j} l\left(\operatorname{Tor}_{\mathrm{pd} M-j-s}(M, N)\right) \\
& =(-1)^{-\mathrm{pd} M+s} \chi(M, N) .
\end{aligned}
$$


Since

$$
-\operatorname{pd} M+s=-(n-\operatorname{dim} M)+\operatorname{dim} N=\operatorname{dim} M+\operatorname{dim} N-n=i,
$$

we are done.

2.3 Corollary. Let $R$ be a Gorenstein ring. Let $M$ be perfect and let $N$ be $C-M$ such that $l\left(M \otimes_{R} N\right)<\infty$. Suppose $M \simeq \check{M}, N \simeq \check{N}$ and $\operatorname{dim} R-\operatorname{dim} M-\operatorname{dim} N$ is odd. Then $\chi(M, N)=0$.

In particular over a regular local ring of $\operatorname{dim} n$, if $R / p$ and $R / q$ are Gorenstein and $n-\operatorname{dim}(R / p)-\operatorname{dim}(R / q)$ is odd, then $\chi(R / p, R / q)=0$.

Proof. By 2.6 we have $\chi(M, N)=-\chi(\check{M}, \check{N})=-\chi(M, N)$. Hence $\chi(M, N)$ $=0$.

2.4 THEOREM. Let $R$ be a Gorenstein ring of $\operatorname{dim} n$. The vanishing conjecture holds in $R \Leftrightarrow$ for any perfect module $M$ and any $C-M$ module $N$ with $\operatorname{dim} M+\operatorname{dim} N=\operatorname{dim} R$, $l\left(M \otimes_{R} N\right)=l\left(M \otimes_{R} \check{N}\right)$.

Proof. $\Rightarrow$ : We consider a prime filtration of $N$. Since $\chi(M, L)=0$ if $\operatorname{dim} M+$ $\operatorname{dim} L<\operatorname{dim} R$, we have

$$
l\left(M \otimes_{R} N\right)=\chi(M, N)=\sum_{p \in \operatorname{Ass}(N)} l\left(N_{p}\right) \chi(M, R / p) .
$$

Similarly,

$$
l\left(M \otimes_{R} \check{N}\right)=\chi(M, \check{N})=\sum_{p \in \operatorname{Ass}(N)} l\left(\check{N}_{p}\right) \chi(M, R / p)=\chi(M, N) .
$$

Since Ass $N=$ Ass $\check{N}$ and $l\left(N_{p}\right)=l\left(\operatorname{Ext}^{\mathrm{ht} p}\left(N_{p}, R_{p}\right)\right)=l\left(\check{N}_{p}\right)$, we have $l\left(M \otimes_{R} N\right)$ $=l\left(M \otimes_{R} \check{N}\right)$.

$\Leftarrow$ : By [D1, 2.2] we can assume $M$ perfect, $Q$ C-M with $l(M \otimes Q)<\infty$, $\operatorname{dim} M+\operatorname{dim} Q<\operatorname{dim} R$. We have to show $\chi(M, Q)=0$. Let $\left\{x_{1}, \ldots, x_{r-1}\right\}$ be an $M$-sequence contained in $\mathrm{Ann}_{R} Q$ such that it is also an $R$-sequence [D1, 2.2]. We consider the exact sequence

$$
0 \rightarrow T \rightarrow\left(R /\left(x_{1}, \ldots, x_{r-1}\right)\right)^{t} \rightarrow Q \rightarrow 0 .
$$

Since $l(M \otimes T)<\infty, \operatorname{dim} M+\operatorname{dim} T=\operatorname{dim} R$, we have by the given condition, $l(M \otimes T)=l(\check{M} \otimes T)$. Hence $\chi(M, Q)=\chi(\check{M}, Q)$. (We notice

$$
\operatorname{Tor}_{i}^{R}(M, Q)=0, \quad i>1,
$$

by (S.L.).)

Let $\left\{y_{1}, \ldots, y_{s-1}\right\}$ be a $Q$-sequence contained in $\mathrm{Ann}_{R} M$ such that it is also an $R$-sequence. We consider the exact sequence

$$
0 \rightarrow N \rightarrow(R / y)^{p} \rightarrow M \rightarrow 0 .
$$

Since $l\left(Q \otimes_{R} N\right)<\infty, \quad M$ perfect, $l(\check{N} \otimes Q)<\infty, \quad \operatorname{dim} N+\operatorname{dim} Q=\operatorname{dim} R$, $l\left(N \otimes_{R} Q\right)=l\left(N \otimes_{R} Q\right)$. Again from (3), applying Hom $(, R)$, we get

$$
0 \rightarrow(R / y)^{p} \rightarrow \check{N} \rightarrow \check{M} \rightarrow 0 .
$$


From (3) and (4) we get

$$
\chi(M, Q)=-\chi(\check{M}, Q) .
$$

From (2) and (5) our result follows.

2.5 COROLlaRY. Let $R$ be a regular local ring of dimension 5. Then the vanishing conjecture holds on $R$.

Proof. By Theorem 2.4, we have to show

$$
l\left(M \otimes_{R} N\right)=l\left(M \otimes_{R} \check{N}\right)
$$

where $M$ is perfect, $N$ is $\mathrm{C}-\mathrm{M}, \operatorname{dim} M+\operatorname{dim} N=\operatorname{dim} R$. We have the following three cases to consider:

Case 1. $\operatorname{dim} N=0$. In this case $\operatorname{dim} M=\operatorname{dim} R$; hence $M \simeq R^{t}, l(M \otimes N)=$ $t l(N)=l(M \otimes \check{N})$.

Case $2 . \operatorname{dim} M=4, \operatorname{dim} N=1$. In this case by killing an $R$-sequence which is also an $M$-sequence of length four contained in $\operatorname{Ann}_{R} N$, we reduce to proving (1) on a complete intersection of $\operatorname{dim} 1$, where $l(M)<\infty$. Since, on a complete intersection of $\operatorname{dim} 1$, the vanishing conjecture holds, we are through.

Case $3 . \operatorname{dim} M=3, \operatorname{dim} N=2$. In this case by killing an $M$-sequence which is also an $R$-sequence of length 3 contained in $\mathrm{Ann}_{R} N$, we reduce to proving (1) on a complete intersection of $\operatorname{dim} 2, l(M)<\infty$. Since, on a complete intersection of $\operatorname{dim} 2$, the vanishing conjecture holds, we are done.

2.6 Proposition. Let $R$ be a Gorenstein ring with $\operatorname{dim} R=3$. Let $M$ be a self-dual module (i.e., $M \simeq \check{M}$ ) of finite length and finite projective dimension. Then for any module $N$ with $\operatorname{dim} N \leqslant 2, \chi(M, N)=0$.

Proof. If $\operatorname{dim} N=1$, we are done by 1.3. So we assume $\operatorname{dim} N=2$. Without any loss of generality we can assume $N$ to be C-M [D1, 2.2]. We consider the exact sequence

$$
0 \rightarrow T \rightarrow\left(R /\left(x_{1}, x_{2}\right)\right)^{t} \rightarrow M \rightarrow 0
$$

where we choose $\left\{x_{1}, x_{2}\right\}$ in such a way that it is both an $N$-sequence and an $R$-sequence; moreover there exists $x \in A$ Ann $N$ such that $\left\{x_{1}, x_{2}, x\right\}$ is an $R$ sequence. This implies $x$ is $T$-regular. Also $T$ is perfect of $\operatorname{dim} 1$ so

$$
\begin{aligned}
\chi(M, N) & =t l\left(N /\left(x_{1}, x_{2}\right) N\right)-l\left(T \otimes_{R} N\right) \\
& =t l\left(N /\left(x_{1}, x_{2}\right) N\right)-l\left(T / x T \otimes_{R / x R} N\right) .
\end{aligned}
$$

Now $R / x R$ is a 2-dimensional Gorenstein ring, hence the vanishing conjecture holds on $R / x R$. Since $T / x T$ is a perfect module of finite length of $R / x R$ and $\operatorname{dim} N=$ $\operatorname{dim}(R / x R), N$ C-M, we have by (2.4)

$$
l\left(T / x T \otimes_{R / x R} N\right)=l\left(T / x T \otimes_{R / x R} \check{N}\right)=l\left(T \otimes_{R} \check{N}\right) .
$$

From (2),

$$
\begin{aligned}
\chi(M, \check{N}) & =t l\left(\check{N} /\left(x_{1}, x_{2}\right) N\right)-l\left(T \otimes_{R} \check{N}\right) \\
& =t l\left(N /\left(x_{1}, x_{2}\right) N\right)-l\left(T \otimes_{R} N\right) \\
& =\chi(M, N) .
\end{aligned}
$$


But we have

$$
\begin{array}{rlrl}
\chi(M, N) & =-\chi(\check{M}, \check{N}) & \text { by }(2.2) \\
& =-\chi(M, \check{N}) \quad(\text { since } M \simeq \check{M}) .
\end{array}
$$

Thus $\chi(M, N)=0$.

Remarks. (1) Definition. A module $M$ on a local ring $R$ is called " $v$-good" if (i) pd $M<\infty$, (ii) given any module $N$ such that $l\left(M \otimes_{R} N\right)<\infty, \operatorname{dim} M+\operatorname{dim} N<$ $\operatorname{dim} R$, then $\chi(M, N)=0$.

Corollary to 2.6. Any perfect self-dual module $M$ on a Gorenstein ring with $\operatorname{dim} M \geqslant \operatorname{dim} R-3$ is $v$-good.

The proof follows by killing an $M$-sequence contained in Ann $N$, where $l(M \otimes N)$ $<\infty, \operatorname{dim} M+\operatorname{dim} N<\operatorname{dim} R$.

(2) In the course of proof we have shown that if $R$ is a 3-dimensional Gorenstein ring, $M$ is a module with $l(M)<\infty$, pd $M<\infty$ and $N$ is C-M with $\operatorname{dim} N=2$, then $\chi(M, \check{N})=\chi(M, N)(2.6(4))$.

2.8 Proposition. Let $R$ be a Gorenstein ring of $\operatorname{dim} n$. Let $N$ be a module with $\operatorname{dim} N=2$ and $M$ a self-dual $C$ - $M$ module such that $l\left(M \otimes_{R} N\right)<\infty, \operatorname{dim} M+$ $\operatorname{dim} N<\operatorname{dim} R$ and $n-\operatorname{dim} M=o d d$. Then $\chi(M, N)=0$.

Proof. Without any loss of generality we can assume $N$ is C-M [D1, 2.2]. We can construct a module $Q$ such that $l(Q \otimes N)<\infty, \operatorname{dim} Q=n-3, \chi(Q, N)=$ $\chi(M, \check{N})$, and

$$
\chi(Q, N)=\chi(M, N) \quad(\operatorname{see}[\mathbf{D 1}])
$$

By killing a maximal $Q$-sequence contained in Ann $N \cap$ Ann $\check{N}$ and using (2),(2.7), we have

$$
\chi(Q, N)=\chi(Q, \check{N})
$$

But

$$
\begin{aligned}
\chi(Q, N) & =-\chi(\check{Q}, N) \quad(\text { by } 2.6) \\
& =-\chi(\check{M}, N) \\
& =-\chi(M, N)
\end{aligned}
$$

From (1)-(3), $\chi(M, N)=0$.

3.

3.1 ThEOREM. Let $R$ be a Gorenstein ring of $\operatorname{dim} n$. The vanishing conjecture holds in $R$ iff given two modules $M, N$ such that $l(M \otimes N)<\infty, \operatorname{pd} M<\infty, \operatorname{dim} M+\operatorname{dim} N$ $<\operatorname{dim} R$, we have $\chi_{1}(M, N)>0$.

Proof. $\Rightarrow$ : Since the vanishing conjecture holds, given $M, N$ as in the theorem, $\chi(M, N)=0$, i.e., $l(M \otimes N)=\chi_{1}(M, N)$. Thus $\chi_{1}(M, N)>0$. (We note that on a local ring the tensor product of two finitely generated modules is nonzero.)

$\Leftarrow$ : By [D1, 2.2], we can assume the case when $M$ is perfect and $N$ is any module such that $l\left(M \otimes_{R} N\right)<\infty, \operatorname{dim} M+\operatorname{dim} N<\operatorname{dim} R$. The proof is by induction on 
$\operatorname{dim} N$. If $\operatorname{dim} N \leqslant 1$, we are done by Proposition 1.3. So we assume $\operatorname{dim} N>1$, and the theorem is true for any module with dimension less than $\operatorname{dim} N$. We take a prime filtration $N$. By the above and (3) in 2.1,

$$
\chi(M, N)=\sum_{\operatorname{dim}(R / p)=\operatorname{dim} N} \chi(M, R / p) \quad \text { (by induction). }
$$

So it will be enough to show $\chi(M, R / p)=0$. Again taking a filtration of $R / p^{n}$ we can show

$$
\chi\left(M, R / p^{n}\right) / l\left(R_{p} / p^{n} R_{p}\right)=\chi(M, R / p) \quad(\text { by induction on } \operatorname{dim} N)
$$

i.e.,

$$
\lim _{n \rightarrow \infty} \frac{\chi\left(M, R / p^{n}\right)}{l\left(R_{p} / p^{n} R_{p}\right)}=\chi(M, R / p)
$$

Now

$$
\chi\left(M, R / p^{n}\right)=l\left(M \otimes R / p^{n}\right)-\chi_{1}\left(M, R / p^{n}\right) .
$$

Since $\operatorname{dim} M+\operatorname{dim}(R / p)<\operatorname{dim} R, \operatorname{dim} M<\operatorname{dim} R-\operatorname{dim}(R / p)=\mathrm{ht} p$. Since $l(M \otimes R / p)<\infty, f(n)=l\left(M \otimes R / p^{n}\right)$ is the Hilbert polynomial of $M$ with respect to $p$ for $n \gg 0$, i.e., for $n \gg 0, f(n)$ is a polynomial whose degree is the dimension of $M$ and hence whose degree is less than ht $p$. On the other hand $g(n)=l\left(R_{p} / p^{n} R_{p}\right)$ is a polynomial in $n$ of degree ht $p$ for $n \gg 0$. Hence

$$
\lim _{n \rightarrow \infty} \frac{l\left(M \otimes R / p^{n}\right)}{l\left(R_{p} / p^{n} R_{p}\right)}=0 .
$$

Thus from (1)-(3),

$$
\chi(M, R / p)=-\lim _{n \rightarrow \infty} \frac{\chi_{1}\left(M, R / p^{n}\right)}{l\left(R_{p} / p^{n} R_{p}\right)} .
$$

Since $\chi_{1}\left(M, R / p^{n}\right)>0$ by assumption, we have

$$
\chi(M, R / p) \leqslant 0 .
$$

Since $M$ is a perfect module, we can find $\left\{x_{1}, \ldots, x_{n}\right\}$ an $R$-sequence such that

$$
0 \rightarrow M \rightarrow\left(R /\left(x_{1}, \ldots, x_{n}\right)\right)^{t} \rightarrow Q \rightarrow 0
$$

is exact. Since pd $M<\infty$, pd $Q<\infty$; also $Q$ is perfect. From (5),

$$
\chi\left(M, \frac{R}{p}\right)+\chi\left(Q, \frac{R}{p}\right)=\chi\left(\left(\frac{R}{\left(x_{1}, \ldots, x_{n}\right)}\right)^{t} \frac{R}{p}\right)=0 .
$$

This forces both $\chi(M, R / p)$ and $\chi(Q, R / p)$ to be 0 . Thus the theorem is established.

3.2 REMARK. We have seen in [D1, 2.2] (reduction to a sublemma) that in order to prove the vanishing conjecture on a Gorenstein ring it is enough to consider the case when $M$ is perfect, $l(M)<\infty, N$ is any other module, with $\operatorname{dim} N<\operatorname{dim} R$. In 3.1 we have shown that by applying induction on $N$, we actually come down to showing $\chi_{1}\left(M, R / p^{n}\right)$ is greater than $0, \operatorname{dim}(R / p)<\operatorname{dim} R$. Since $l(M)<\infty$ we have 
$p^{n} M=0$, for $n \gg 0$. Now from $0 \rightarrow p^{n} \rightarrow R \rightarrow R / p^{n} \rightarrow 0$ we get $\chi_{1}\left(M, R / p^{n}\right)=$ $\chi\left(M, p^{n}\right)$ (since $M \stackrel{\sim}{\rightarrow} M \otimes R / p^{n}$ for $\left.n \gg 0\right)$. Thus $\chi_{1}\left(M, R / p^{n}\right)>0$ iff $\chi\left(M, p^{n}\right)>$ 0 . Hence we have proved the following:

THEOREM. On a Gorenstein ring of $\operatorname{dim} n$, the nonvanishing conjecture implies the vanishing conjecture.

We note here that for our result to be true we need only the nonvanishing conjecture to be true for a pair of modules, one of which is perfect.

We now state two results without proof and we refer the reader to [D2, (1.8), Corollaries 5 and 6] for the proofs.

3.3 Proposition. Let $R$ be a Gorenstein ring of dimension 3 and $\operatorname{ch} R=p>0$. Let $M$ be a perfect module of finite length. Then $M$ is v-good iff given $N$ with $\operatorname{dim} N=3$, $\chi(M, N)>0$.

3.4 Proposition. Let $R$ be a complete ramified regular local ring of $\operatorname{dim} 5$ and let $p$ denote a generator of the maximal ideal of the d.v.r. $V$ contained in $R$ such that $V / p V \simeq R / m$, where $m$ is the maximal ideal of $R$. Let $M$ and $N$ be two modules over $R$ such that (i) $l(M \otimes N)<\infty$, (ii) $\operatorname{dim} M+\operatorname{dim} N=5$, (iii) $p M=0, p$ is a n.z.d. on $N$ and $N$ is perfect. Then $\chi(M, N)>0$.

4.

4.1. Let $R$ be a regular local ring and $I$ an ideal of $R$. Let $C_{I}$ be the set of finite free complexes over $R$ with homology killed by $I^{n}$ for some $n>0$. Let $G_{I}=$ free abelian group on elements of $C_{I}$ modulo the subgroup generated by (i) exact complexes, (ii) $C_{2}-C_{1}-C_{3}$, where $0 \rightarrow C_{1} \rightarrow C_{2} \rightarrow C_{3} \rightarrow 0$ is an exact sequence of complexes.

Proposition. $G_{I}$ is generated by free resolutions of modules $M$ with $I^{n} M=0$ for some $n>0$.

Note. Fossum, Griffith and Iverson [F-G-I] have proved the case when $I=m$, the maximal ideal of $R$.

Proof. Let $C$ be any finite free complex. We assume $H_{0}(C) \neq 0$ (otherwise we can split off an acyclic free complex from $C$ and start with truncated $C$ ). Let $M=H_{0}(C)$. We have a projective resolution of $M, K_{0}: 0 \rightarrow R^{t_{n}} \rightarrow \cdots \rightarrow R^{t_{0}} \rightarrow 0$, with $H_{0}\left(K_{0}\right)=M$.

Claim. We can construct a finite free complex $D_{0}$ such that the number of nonzero homologies of $D_{0}$ is less than that of $C$.

Proof. Since components of $C$ are free and $K_{0}$ is exact we have a map $\phi: C \rightarrow K_{0}$. We construct the cone " $D_{0}$ " of $C$ by $K_{0}$ via $\phi$, i.e., a complex $D_{0}^{\text {" where }}$

$$
\left(D_{0}^{\prime \prime}\right)_{n}=\left(K_{0}\right)_{n}+C_{n-1}
$$

and $d_{n}^{\prime \prime}:\left(D_{0}^{\prime \prime}\right)_{n} \rightarrow\left(D_{0}^{\prime \prime}\right)_{n-1}$ is given by

$$
d_{n}^{\prime \prime}(x, y)=\left(d_{n}^{\prime}(x)+\phi_{n-1}(y),-d_{n-1}(y)\right) .
$$


Hence we have the exact sequence of complexes

$$
0 \rightarrow K_{0} \rightarrow D_{0}^{\prime \prime} \rightarrow C_{-1} \rightarrow 0
$$

where $\left(C_{-1}\right)_{n}=C_{n-1}$. The last few terms of the long exact sequence of homologies obtained from (1) are as follows:

$$
\rightarrow H_{2}\left(C_{-1}\right) \rightarrow H_{1}\left(K_{0}\right) \rightarrow H_{1}\left(D_{0}^{\prime \prime}\right) \rightarrow H_{1}\left(C_{-1}\right) \rightarrow H_{0}\left(K_{0}\right) \rightarrow H_{0}\left(D_{0}^{\prime \prime}\right) \rightarrow 0 .
$$

It is easy to see $H_{0}\left(D_{0}^{\prime \prime}\right)=0$. Since $H_{1}\left(C_{-1}\right) \stackrel{\sim}{\rightarrow} H_{0}\left(K_{0}\right)$ and $H_{i}\left(K_{0}\right)=0$ for all $i \geqslant 1$, we have $H_{1}\left(D_{0}^{\prime \prime}\right)=0$. We also note $H_{i}\left(D_{0}^{\prime \prime}\right)=H_{i}\left(C_{-1}\right)=H_{i-1}(C)$ for $i \geqslant 2$. Let $t=\min \left\{j: H_{j}\left(D_{0}^{\prime \prime}\right) \neq 0\right\}$. Denoting the $i$ th component of $D_{0}^{\prime \prime}$ by $F_{i}$ we see that

$$
F_{t+1} \stackrel{\psi_{t+1}}{\rightarrow} F_{t} \stackrel{\psi_{t}}{\rightarrow} F_{t-1} \stackrel{\psi_{t-1}}{\rightarrow} \cdots \rightarrow F_{1} \rightarrow F_{0} \rightarrow 0
$$

and that $D_{0}^{\prime \prime}$ splits off $0 \rightarrow \operatorname{Im} \psi_{t} \rightarrow F_{t-1} \rightarrow \cdots F_{1} \rightarrow F_{0} \rightarrow 0$ which is acyclic, and we have a new free complex $D_{0}$ given by

$$
\rightarrow F_{i+1} \rightarrow F_{i} \rightarrow \cdots \rightarrow F_{t+1} \rightarrow F_{t}^{\prime \prime} \rightarrow 0
$$

where $F_{t}^{\prime \prime}=\operatorname{ker} \psi_{t}$.

We easily check that the number of nonzero homologies of $D_{0}$ is less than that of $C$. Thus the claim is proved.

Now continuing the above procedure a finite number of times, we arrive at a finite free complex $D$ with $H_{0}(D) \neq 0, H_{i}(D)=0$ for all $i>0$, and in $G_{I}, C$ is generated by these $K_{i}$ 's and $D$. Thus $G_{I}$ is generated by free resolutions of modules $M$ with $I^{n} M=0$ for some $n>0$.

4.2 THEOREM. Let $R$ be a ring of formal power series over a field or d.v.r. Let $C_{I}=$ finite free complexes over $R$ with homology killed by $I^{n}$ for $n>0$ where $I$ is an ideal of $R$. If $I$ and $J$ are two ideals of $R$ such that $I+J$ is primary to $m$, the maximal ideal of $R$, we have a map $\theta_{I, J}: G_{I} \otimes G_{J} \rightarrow Z$ via $\left[C_{1}\right] \otimes\left[C_{2}\right] \rightarrow \chi$ (total complex $\left(C_{1} \otimes C_{2}\right)$ ) (i.e., alternate sum of length of homologies). If $\operatorname{dim}(R / I)+\operatorname{dim}(R / J)<$ $\operatorname{dim} R$, then $\theta_{I, J} \equiv 0$.

Proof. By (4.1) it is enough to show that if $C$ is a finite free resolution of a module $M$ with $I^{n} M=0$ for some $n>0$ and if $D$ is a finite free resolution of a module $N$ with $J^{t} N=0$ for some $t>0$, then $\chi(C \otimes D)=0$. But $\chi(C \otimes D)=$ $\chi(M, N)$ and since $\operatorname{dim} M+\operatorname{dim} N<\operatorname{dim} R, \chi(M, N)=0$ by [S, Chap. V, Theorem 1, Lemma].

Thus $\theta_{I, J} \equiv 0$.

\section{REFERENCES}

[A-B] M. Auslander and D. Buchsbaum, Codimension and multiplicity, Ann. of Math. (2) 68 (1958), 625-657.

[C-E] H. Cartan and S. Eilenberg, Homological algebra, Chap. XVI, Princeton Univ. Press, Princeton, N. J., 1956.

[D1] S. P. Dutta, Weak linking and intersection multiplicity, J. Pure Appl. Algebra (to appear).

[D2] __ Frobenius and multiplicities, J. Algebra (to appear).

[F] H. B. Foxby, Intersection multiplicities of modules (preprint).

[F-F-I] R. M. Fossum, H. B. Foxby and B. Iverson, A characteristic class in algebraic $k$-theory (preprint). 
[H] M. Hochster, Conference on Commutative Algebra, Lecture Notes in Math., vol. 311, SpringerVerlag, Berlin and New York, 1973, pp. 120-152.

[L] S. Lichtenbaum, On the vanishing of Tor in regular local rings, Illinois J. Math. 10 (1966), 220-226.

[M] M. P. Mallaivain-Brameret, Une remarque sur les anneaux locaux réguliers, Sém. Dubreil-Pisot (Algèbre et Théorie des Nombres), 1970/71, $\mathrm{n}^{\circ} 13$.

[P-S] C. Peskine and L. Szpiro, Syzygies et multiplicités, C. R. Acad. Sci. Paris Ser. A-B 278 (1974), $1421-1424$.

[S] J. P. Serre, Algèbre locale. Multiplicités, Lecture Notes in Math., vol. 11, Springer-Verlag, Berlin and New York, 1975.

Department of Mathematics, University of Pennsylvania, Philadelphia, Pennsylvania 19104 